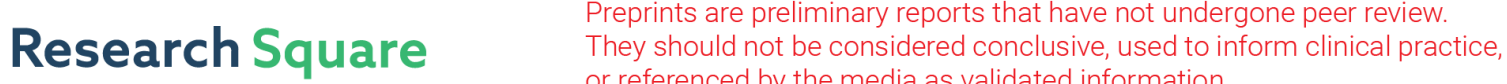 or referenced by the media as validated information. \\ Ensemble EfficientNet and Mixed Convolution for Three-class brain MR image classification
}

Jagadeesh Kakarla ( $\nabla$ jagadeeshk@iiitdm.ac.in )

IIITDM Kancheepuram: Indian Institute of Information Technology Design and Manufacturing

Kancheepuram

Bala Venkateswarlu Isunuri

Indian Institute of Information Technology Design and Manufacturing Kancheepuram

\section{Research Article}

Keywords:

Posted Date: March 8th, 2022

DOI: https://doi.org/10.21203/rs.3.rs-1076068/v1

License: (1) This work is licensed under a Creative Commons Attribution 4.0 International License.

Read Full License 


\title{
Ensemble EfficientNet and Mixed Convolution for Three-class brain MR image classification
}

\author{
Isunuri Bala Venkateswarlu • Jagadeesh Kakarla*
}

Received: date / Accepted: date

\begin{abstract}
The classification of brain tumor images is the prevalent task in computer-aided brain tumor diagnosis. Recently, three-class classification becomes a superlative task in brain tumor classification. The existing models are fine-tuned for a single dataset, and hence they may exhibit displeasing results on other datasets. Thus, there is a need for a generalized model that can produce superior performance on multiple datasets. In this paper, we have presented a generalized model that produces similar results on two datasets. We have proposed an ensemble EfficientNet and mixed convolution model to perform a three-class classification. We have devised a mixed convolution to enhance the feature vector extracted from pre-trained EfficientNet. The mixed convolution consists of two blocks namely separable convolution and residual convolution blocks. We have utilized a gaussian dropout layer before the softmax layer to avoid model overfitting. In our experiments, two publicly available datasets are considered for the evaluation of the proposed model. We have achieved an accuracy of $97.65 \%$ and $95.84 \%$ on BTDS and CPM datasets, respectively. The proposed model outperforms existing pre-trained models and state-of-the-art models in vital metrics.
\end{abstract}

\section{Introduction}

Brain cancer is one of the most life-threatening diseases and becomes the deadliest hazard in the world. In general, brain cancer can arise from the cancerous cells of the brain tumor. The abnormal growth of brain tissues

\footnotetext{
Jagadeesh Kakarla

Indian Institute of Information Technology, Design and Manufacturing, Kancheepuram, Chennai, India

E-mail: jagadeeshk@iiitdm.ac.in
}

is referred to as a brain tumor and can be malignant or benign. The malignant tumor is cancerous and incurable with medication that needs surgery. In general, medical imaging has become a dominant technique for brain tumor diagnosis. The magnetic resonance (MR) images manifest detailed pictures of brain cells. Thus, several neurologists recommend MR images for the detection of brain tumors. The researchers are motivated for automatic brain tumor diagnosis due to several reasons. The primary concerns for automation are as follows.

- Reduction of human intervention: The manual inspection of each patient has become a tedious task for neurologists due to the huge number of cases. It can be reduced using automatic diagnosis tools.

- Helps early detection: The early detection of brain tumors can reduce serious consequences with timely medication. It also helps the physician to improve the quality of diagnosis and the patient's health [9].

The computer-aided brain tumor diagnosis can be considered as a second opinion by the physicians while making the final decision [1]. The tumor image classification and tumor region segmentation are the major tasks of the automatic brain diagnosis tools. However, tumor classification is the prevelant task in the computer-aided tumor diagnosis. There are two types of classification algorithms based on number of classes, namely binary and multi-class. The binary classification of brain tumor images is also known as brain tumor detection. It mainly detects whether the given input brain tumor image is healthy or not. In literature, several proposals are reported on brain tumor detection as follows.

Zhang et al. [2] have utilized fractal-dimension estimation along with minkowski-bouligand method for pathological brain detection. Nayak et al. [3] have de- 
vised extreme learning machine and combined with a modified sine-cosine algorithm. Kaur et al. [4] have defined fisher criterion as a fitness function and a feature fusion as optimization for classification of brain tumor. Shankar et al. [5] have employed an adaptive neurofuzzy based interface system for the segregation of brain MR images into malignant or benign. These methods are evaluated on the publicly available dataset namely whole brain atlas (WBA) [6]. On the other hand, some of them have used private datasets for performance analysis. The machine learning techniques are effective for smaller datasets. Hence, most of the authors have employed machine learning for brain tumor detection.

Recently, deep learning has become popular for image classification tasks. There is a scant number of proposals observed on binary classification. Siyuan et al. [7] have devised a pre-trained AlexNet for the brain pathology detection. Another deep transfer learning approach has presented by Mohammad et al. [8] for classification of brain abnormality. Nowadays, brain tumor type detection is a critical task due to the complex characteristics of tumors. There are more than 120 types of primary tumors from the brain and central nervous system. However, there are three familiar brain tumors as follows [9].

1. Meningioma tumor forms on cells of the brain and spinal cord just under the skull area.

2. Pituitary tumor effects in or around the pituitary gland portion of the brain.

3. Glioma is a type of tumor that begins in gluey supportive glial cells. There are several sub types of glioma and some of them are as follows.

- Glioblastoma starts from star-shaped cells in the brain called astrocytes.

- Oligodendroglioma effects oligodendrocytes in the brain and spinal cord.

- Astrocytoma can occur in the brain or spinal cord.

In general, the characteristics of a tumor can be differentiated using various properties such as texture, location, shape, and size. The texture and location are the primary feature to be employed for the segregation of meningioma and pituitary tumors. On the other hand, glioma tumors can be identified by the shape and size of the tumors. Thus, multi-class classification of brain tumor becomes a critical research task due to the distinct properties of tumors [10]. It motivated us to work in this direction, and hence we have proposed an ensemble model for the three-class classification of brain MR images.

The remaining paper has organized as follows; Section 2 presents the review of three-class brain tumor classification. Section 3 describes methodogy used in the work. Section 4 reports performance analysis of the proposed model, and Section 5 concludes findings of the work.

\section{Related work}

Cheng et al. [11] has instigated a three-class brain MR image classification using a feature extraction framework. They have published a dataset with 3064 images and have achieved $94.68 \%$ precision. Several researchers addressed the three-class classification using machine learning as follows. Gumai et al. [12] have employed a principal component analysis (PCA) based normalized GIST descriptors for feature extractor. Authors have attained an accuracy of $94.23 \%$ regularized extreme learning machine. In general, hand-crafted feature extraction is the critical step in traditional machine learning. It mainly depends on dataset and researcher feature selection skills. CNN utilizes automatic feature extraction with a sequence of convolution layers. However, the selection of networks is challenging in CNNbased classification. Anaraki et al. [13] have utilized the best convolution neural network (CNN) structure using a genetic algorithm and have achieved $94.20 \%$ accuracy. The existing proposals have encountered a low accuracy due to limited hand-crafted features.

Later, few authors adopted transfer learning to improve performance. Swati et al. [14] have fine-tuned the pre-trained model for the classification of brain tumor images. The authors have demonstrated the transferability of learning using a pre-trained VGG19 model. The VGG19 has been used to extract generic features, and six sequential blocks have been utilized for domainspecific feature extraction. They have achieved a classification accuracy of $94.82 \%$ with a block-wise finetuning strategy. Ghassemi et al. [15] have designed a generative adversarial network to extract robust features from various MR image datasets. A six-layer deep neural network classifier has been employed for threeclass brain tumor classification. They have achieved 95.6\% accuracy using pre-trained GAN with data augmentation. In this case, the source domain is Imagenet, and the destination domain is brain image knowledge. Thus, simple transfer learning is not proficient due to a mismatch between source and destination domains. These deep learning methods are computationally expensive due to a huge number of trainable parameters. Thus, optimization of deep networks has become a challenging task in image classification.

Recently, Deepak et al. [16] have coined ensemble learning for three-class classification of brain tumor. A pre-trained GoogleNet is used to extract features from 
the given brain MR image. Then, the features are processed using a support vector machine classifier and have achieved $97.10 \%$ accuracy. However, authors have reported the results on only one brain tumor dataset. The existing models are fine-tuned for a single dataset, and hence there is a need for generalized models. It motivated us to work in this direction for an efficient threeclass brain tumor image classification model. Hence, we have proposed an ensemble EfficientNet and mixed convolution for three-class classification. The proposed work has focused on the following objectives.

1. Design of optimized network for three-class classification of brain MR images.

2. Implementation of a generalized network that produces similar performances on two contrast-enhanced T1 weighted MR image datasets.

3. Minimize the number of trainable parameters.

\section{Description of datasets}

We have considered two publicly available brain tumor datasets for the three-class brain tumor classification. Firstly, Cheng et al. [11] have published a brain tumor dataset (BTDS) having 3064 T1-weighted contrast-enhanced MR images [17]. BTDS has enriched with magnetic resonance images with three types of tumors: meningioma, glioma, and pituitary. It can be used for both classification and segmentation. We have performed the basic preprocessing operations such as scaling and normalization. Initially, we have performed min-max normalization on the BTDS dataset. Then, the size of the image is scaled to $(256,256,3)$ from $(512,512,3)$ for computational efficiency.

Recently, the Computational precision medicine (CPM) dataset [18] has been published as a part of the MICCAI 2019 challenge which consists of multi-institutional paired radiology scans. These scans are skull-stripped and interpolated to the same size as $(240,240,120)$. CPM training data contains MR scans of 222 subjects and are classified into three sub-types: glioblastoma, oligodendroglioma, and astrocytoma. We have considered $\mathrm{T} 1$ weighted contrast-enhanced 3D subjects for the experiments. Initially, each subject is normalized using min-max and then each 3D subject is converted into 2D slices. A manual inspection of slices has been performed to find the number of slices and dimensions of each slice. We have extracted 40 slices (20 axial, 20 sagittal, and 20 coronal) from each subject to generate the $2 \mathrm{D}$ CPM dataset. Finally, each 2D slice is resized as $(160,160$, 3 ) to achieve optimal computational cost. Table 1 lists out complete details of considered datasets.

\section{Methodology}

The deep convolutional networks (DeepConvNet) become effective and successful models for image classification. The traditional convolutional neural network $(\mathrm{CNN})$ consists of various layers, including input, hidden, and classification. The input layer allows reading input images with optional pre-processing steps like scaling, zooming, etc. Then, the hidden layer that consists of several sub-layers performs feature extraction. Convolution and pooling are the common sub-layers that are used for feature extraction and dimensionality reduction, respectively. Finally, the classification layer performs probability computation for each class and allows for the segregation of the given input image into one of the classes. If $\mathbb{F}_{i}($.$) is the function that defines the$ $i^{\text {th }}$ layer of convolution network, then a convolutional neural network model $\mathbb{N}$ can be defined using Eq. 1 . Where, $\mathbb{X}^{i-1}$ is input tensor at layer $i$ having tensor shape $<W_{j}, H_{j}, C_{j}>, n$ is number of layers and $\odot$ represents sequential model generation operator.

$$
\mathbb{N}=\odot_{i=1}^{n} \mathbb{F}^{i}\left(X_{<W_{j}, H_{j}, C_{j}>}^{i-1}\right)
$$

DeepConvNets are designed with a large number of sub-layers to achieve better accuracy. The complexity of the model increases with the number of sub-layers in the hidden layer. Moreover, DeepConvNets are often over-parameterized and suffer from vanishing gradient problems due to an increase in depth of network [19]. Kaiming et al. [20] have introduced residual connections to solve vanishing gradients. They have designed the ) residual networks with different depths ranging from ResNet-18 to ResNet-200. Howard et al. [21] have presented a variant of deep networks with increased channel depth referred to as MobileNet. Later, Mingxing et al. [19] have presented a family of EfficientNet models using model scaling. The authors have systematically studied model scaling in depth/width/resolution. They have identified an effective component efficient model as the base model i.e. EfficientNetB0. The building block for the EfficientNetB0 is inverted bottleneck MBConv with a squeeze and excitation optimization. A sequence of seven MBConv blocks is used to design the EfficientNetB0 model. The authors have presented the family of eight optimal EfficientNet models using model scaling of depth/width/resolution. The proposed model has considered an ensemble of EfficientNet model with a mixed convolution block. The detailed implementation of the proposed model is as follows. 
Table 1: Summary of datasets

\begin{tabular}{|c|c|c|c|}
\hline Dataset & Size & \#Images & Classes \\
\hline BTDS & $(256 \times 256 \times 3)$ & 3064 & $\begin{array}{l}\text { Meningioma (708), } \\
\text { Glioma (1426), } \\
\text { Pituitary (930) }\end{array}$ \\
\hline CPM & $(160 \times 160 \times 3)$ & 8880 & $\begin{array}{c}\text { Glioblastoma }(2200), \\
\text { Oligodendroglioma }(5320), \\
\text { Astrocytoma }(1360)\end{array}$ \\
\hline
\end{tabular}

\subsection{Transfer learning with EfficientNet}

Transfer learning is the widely used technique in deep learning to solve new problems using knowledge sharing. The major advantages of transfer learning are improvement of performance and fast training. Thus, we have adopted pre-trained EfficientNet as the backbone model for the proposed model. EfficientNetB0 is the basic model for the family of EfficientNets. All the other EfficientNets are scaled from the EfficientNetB0 with different compound coefficients [19]. We have analyzed the models and observed the following points.

1. EfficientNetB0 reports least top-5 accuracy (93\%) with 5.3M parameters and EfficientNetB7 reports highest top-5 accuracy (97\%) with 66M parameters.

2. EfficientNetB2 achieves moderate top-5 accuracy (95\%) with only $12 \mathrm{M}$ parameters.

3. EfficientNetB2 exhibits $2 \%$ improvement than EfficientNetB0 and 2\% lesser performance than EfficientNetB7 in top-5 accuracy.

It motivated us to consider EfficientNetB2 as a backbone model for achieving better performance with optimal parameters. EfficientNetB2 also uses seven MBConv blocks as EfficientNetB0. However, there is a change in the number of filters in each MBConv block.

Table 2: EfficientNetB2 network

\begin{tabular}{clcc}
\hline Stage & Operator & \multicolumn{2}{c}{ Output dimension } \\
& & for BTDS & for CPM \\
\hline 1 & Input \& stem & $(256,256,3)$ & $(160,160,3)$ \\
2 & MBConv1 & $(128,128,16)$ & $(80,80,16)$ \\
3 & MBConv2 & $(64,64,24)$ & $(40,40,24)$ \\
4 & MBConv3 & $(32,32,48)$ & $(20,20,48)$ \\
5 & MBConv4 & $(16,16,88)$ & $(10,10,88)$ \\
6 & MBConv5 & $(16,16,120)$ & $(10,10,120)$ \\
7 & MBConv6 & $(8,8,208)$ & $(5,5,208)$ \\
8 & MBConv7 & $(8,8,352)$ & $(5,5,352)$ \\
9 & TopConv & $(8,8,1408)$ & $(5,5,1408)$ \\
\hline
\end{tabular}

The proposed model takes dimensions of $(256,256$, $3)$ and $(160,160,3)$ for BTDS and CPM datasets, respectively. It produces feature vectors having incremental filters as 16, 24, 48, 88, 120, 208, 352 at each stage. The detailed output dimensions for both datasets are listed in Table 2. Final convolution outputs feature vector having dimension of $(w, h, 1408)$. Here, $w$ and $h$ denotes width and height of the image.

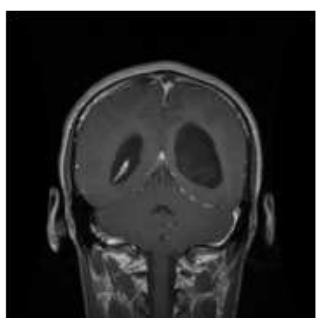

(a) BTDS slice

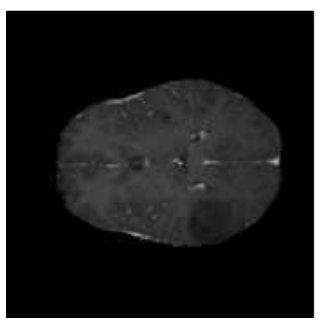

(b) CPM slice
Fig. 1: Sample brain MR images
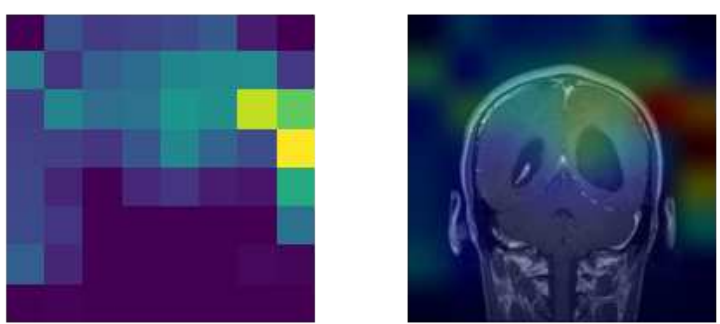

(a) Class prediction map of BTDS slice
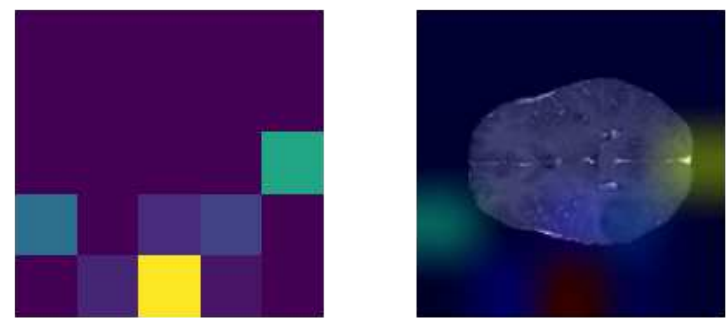

(b) Class prediction map of CPM slice

Fig. 2: Pre-trained EfficientNet results 


\subsection{Mixed convolution block}

In our experiments, we have observed that the pretrained EfficientNet2 is unable to recognize semantic features of the brain. A sample brain MR images from BTDS and CPM datasets as shown in Fig. 1 (a) and (b), respectively. The class prediction map visualizes the feature map of the given image before classification layers. The model is efficient if more brain regions are captured in the class prediction map. The pretrained EfficientNetB2 has captured more amount of non-brain regions in its class prediction map and can be observed from Fig. 2. However, very few amounts of the non-brain region have been included in skull stripped brain MR image as shown in Fig. 2 (b). The reason behind this behavior is due to the Imagenet dataset of the pre-trained model. The knowledge from the Imagenet dataset is not directly suitable for medical image datasets. It needs re-convolution of feature map to improve feature map obtained from the pre-trained model. Thus, we have proposed a mixed convolution block to enhance the features obtained from transfer learning. This block is named as Mixed Convolution block as it uses both separable convolution and basic convolution in two different paths. The details of the proposed mixed convolution block are as follows.

1. Separable convolution block: A sequence of two separable convolution layers with average pooling has been used in this block. Each separable convolution uses 704 filters with $(1 \times 1)$ kernel. Similarly, each average pooling includes a pooling size of $(2 \times 2)$. The final output is obtained with LeakyReLU having $\alpha=0.3$ as shown in Fig. 3 (a). This block takes the role of domain-specific feature extraction by capturing the semantic features of the brain.

2. Residual convolution block: This block consists of three convolutions with 704 filters and $(1 \times 1)$ kernel. The vanishing gradient is one of the problems in deep networks. Thus, we have devised the residual connection to avoid vanishing gradient. The final output is produced with LeakyReLU having $\alpha=0.3$ as shown in Fig. 3 (b). This block supports separable convolution block in feature enhancement.

\subsection{Proposed ensemble model}

The proposed model has adopted an ensemble of transfer learning and mixed convolution for three-class brain tumor classification. The detailed steps for the implementation of the proposed model are as follows.

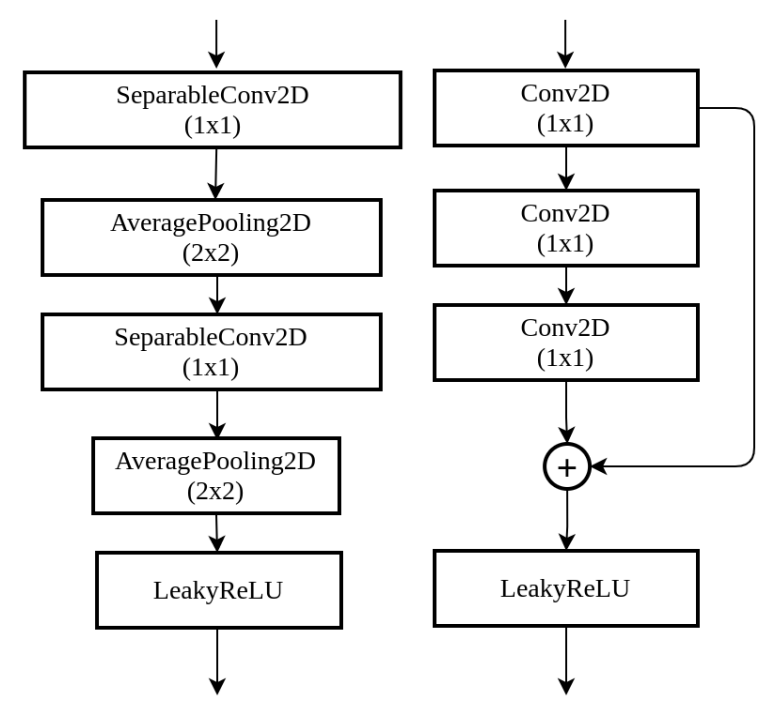

(a) Separable Convolution(b) Residual Convolution block block

Fig. 3: Proposed convolution blocks

1. Transfer learning: Given input image $\mathbf{I}$ is first poccessed with EfficientNetB2 to extract feature vector FV. The pre-trained EfficientNetB2 transforms shape of the input image is $(\mathrm{W}, \mathrm{H}, 3)$ to $(\mathrm{w}, \mathrm{h}$, 1408). This feature vector is re-convoluted using ensembeled mixed convolution block.

2. Mixed convolution: The feature vector FV obtained from transfer learning is re-convoluted using mixed convolution. FV is processed by separable and residual convolution blocks to produce SFV and RFV vectors, respectively. Then, the global average pooling is applied to each feature vector to produce one-dimensional vector. Here, global average pooling $\mathbf{G}(z)$ is used instead of flatten layer and can be defied for the given $3 \mathrm{D}$ vector $\mathbf{V}$ as follows.

$\mathbf{G}(z)=\frac{\sum_{j=1}^{y} \sum_{i=1}^{x} \mathbf{V}(i, j, z)}{x * y}$

If $\mathbf{G}_{\mathbf{S F V}}$ and $\mathbf{G}_{\mathbf{R F V}}$ are the output vectors after global average pooling, then the final feature vector F can be obtained as follows.

$\mathbf{F}_{\mathbf{i}}=\frac{\mathbf{G}_{\mathrm{SFV}}+\mathbf{G}_{\mathrm{RFV}}}{2}$

3. Classification: The feature vector $\mathbf{F}$ obtained from ensemble EfficientNetB2 and mixed convolution is processed using two dense layers. The first dense layer consists of 320 neurons activated with ReLU function. Then, a gaussian dropout with a dropout rate of 0.5 is applied to reduce overfitting problems. 


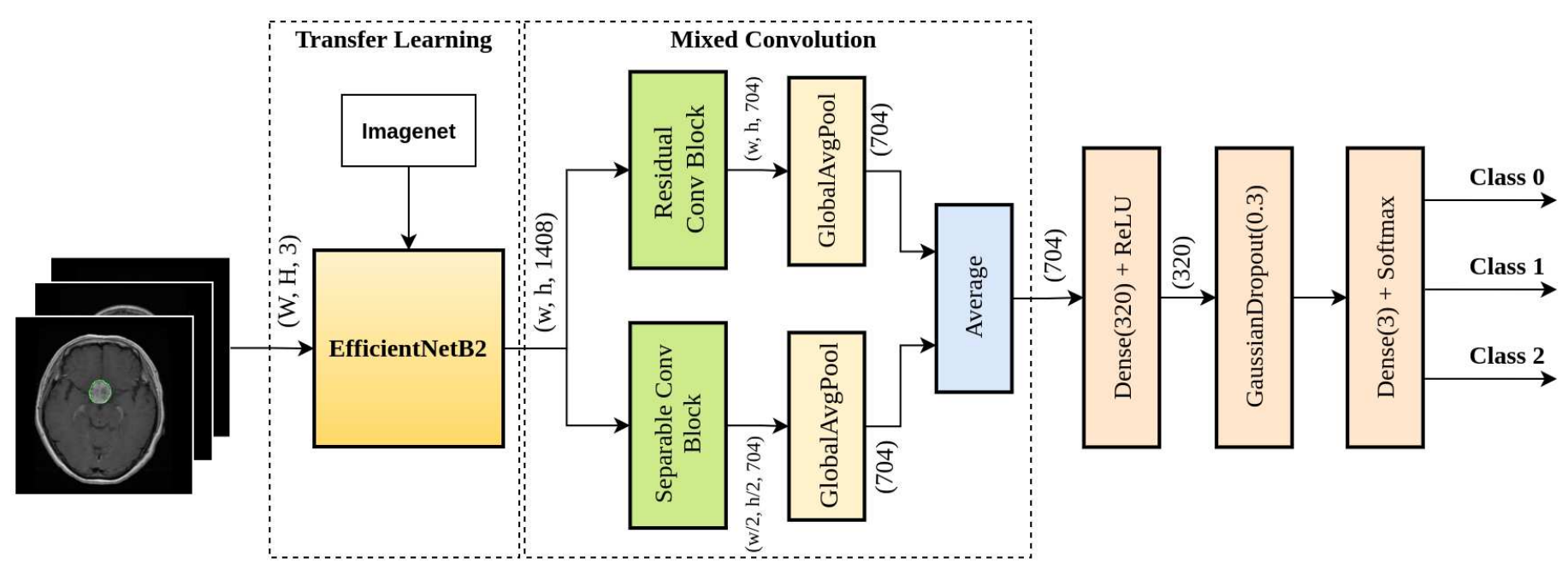

Fig. 4: Block diagram of the proposed model

The second dense layer with 3 neurons having softmax activation is used to produce the final classification result $C$ as follows.

$$
C=\operatorname{Dense}(G D(\operatorname{Dense}(\mathbf{F})))
$$
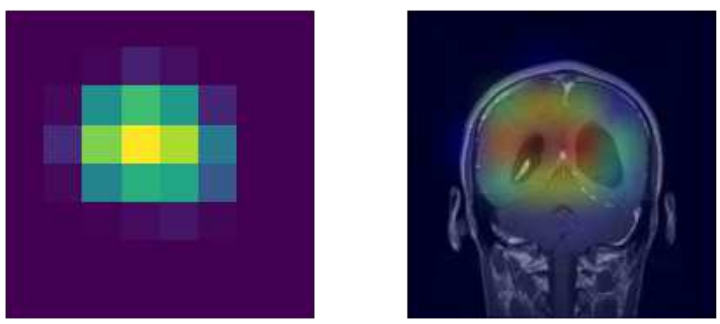

(a) Class prediction map of BTDS slice
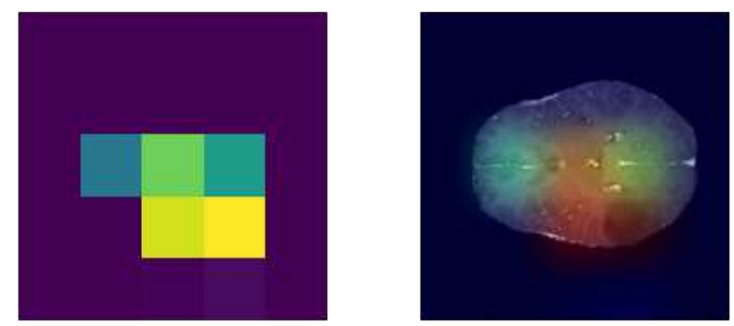

(b) Class prediction map of CPM slice

Fig. 5: Proposed model results

Fig. 5 visualizes the class prediction map of BTDS and CPM slices obtained by the proposed ensemble model. It can be observed that our model adheres to most of the brain regions in its class prediction map. The proposed model exhibits similar performance in both cases i.e. brain MR image with and without the skull. Moreover, our model achieves a high probability at the tumor region in its class prediction map.

\section{Results and discussion}

Firstly, we have included the experimental setup details, and description of datasets. Then, the performance of proposed model has discussed in this section. The proposed ensemble EfficientNet and mixed convolution (EEMC) model has evaluated on two publicly available datasets: BTDS [17] and CPM [18]. For the evaluation, we have considered both pre-trained models and stateof-the-art model. MobileNet, InceptionResNet, GoogleNet, ResNet, and EfficientNet are the popular pre-trained models and hence they are considered for the evaluation. Similarly, we have compared our results with state-of-the-art models including Anaraki et al. [13] and Deepak et al. [16]. Accuracy, precision, recall, and jaccard similarity are the vital performance metrics for classification and hence we have considered for numerical evaluation.

\subsection{Experimental setup}

The proposed ensemble model and other pre-trained models are implemented using Python and Keras. Google CoLab with GPU has been utilized to conduct our experiments. Adam is one of the widely used optimizer for deep neetworks. The implementation of Adam optimizer is easy and exhibits faster convergence. Hence, we have trained our model using Adam optimizer with 0.0003 as initial learning rate. Table 3 lists out the complete hyperparameter settings used for the experiments. 
Table 3: Hyperparameter settings

\begin{tabular}{|c|c|}
\hline Hyperparameter & Value \\
\hline Loss function & Sparse categorical crossentropy \\
Optimizer & Adam \\
Initial learning rate & 0.0003 \\
Batch size & 2 \\
Number of epochs & 50 \\
\hline
\end{tabular}

\subsection{Evaluation of proposed model on BTDS dataset}

The performance evaluation of the proposed EEMC model on the BTDS dataset has presented in this section. Our model achieves maximum accuracy with in 50 epochs and Fig. 6 shows the training and validation accuracy. We have evaluated our model using stratified five-fold cross-validation and the perfomance in each fold has listed in Table 4. Our model has exhibited the best performance in the second fold with $98.21 \%$ accuracy. It is observed that our model has experienced the least performance in the third fold. Further, our model achieves a mean accuracy of $97.65 \%$ with a 0.59 deviation.

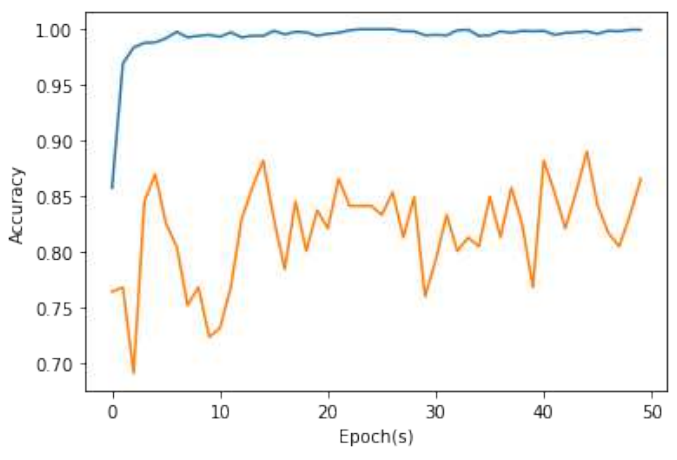

Fig. 6: Training vs validation accuracy

Table 4: Stratified five-fold cross-validation

\begin{tabular}{|c|cccc|}
\hline Fold & Accuracy & Precision & Recall & Jaccard \\
\hline 1 & 98.04 & 97.92 & 97.52 & 95.56 \\
2 & 98.21 & 97.65 & 98.11 & 95.85 \\
3 & 96.74 & 96.03 & 96.77 & 93.07 \\
4 & 97.88 & 97.58 & 97.59 & 95.31 \\
5 & 97.39 & 97.46 & 96.69 & 94.28 \\
\hline Mean & 97.65 & 97.33 & 97.34 & 94.81 \\
Stddev & 0.59 & 0.74 & 0.6 & 1.14 \\
\hline
\end{tabular}

We have analyzed wrong prediction cases using class prediction maps as shown in Fig. 7. Our model detects the location of the tumor while predicting the tumor type which can be observed from the last row of Fig. 7. This shows our model preserves more semantic features due to the mixed convolution block. However, the proposed model results in the wrong prediction when there is more amount of non-brain regions such as the skull. The first three rows of Fig. 7 represents wrong predictions of the tumor and it is due to existence of more non-brain regions.
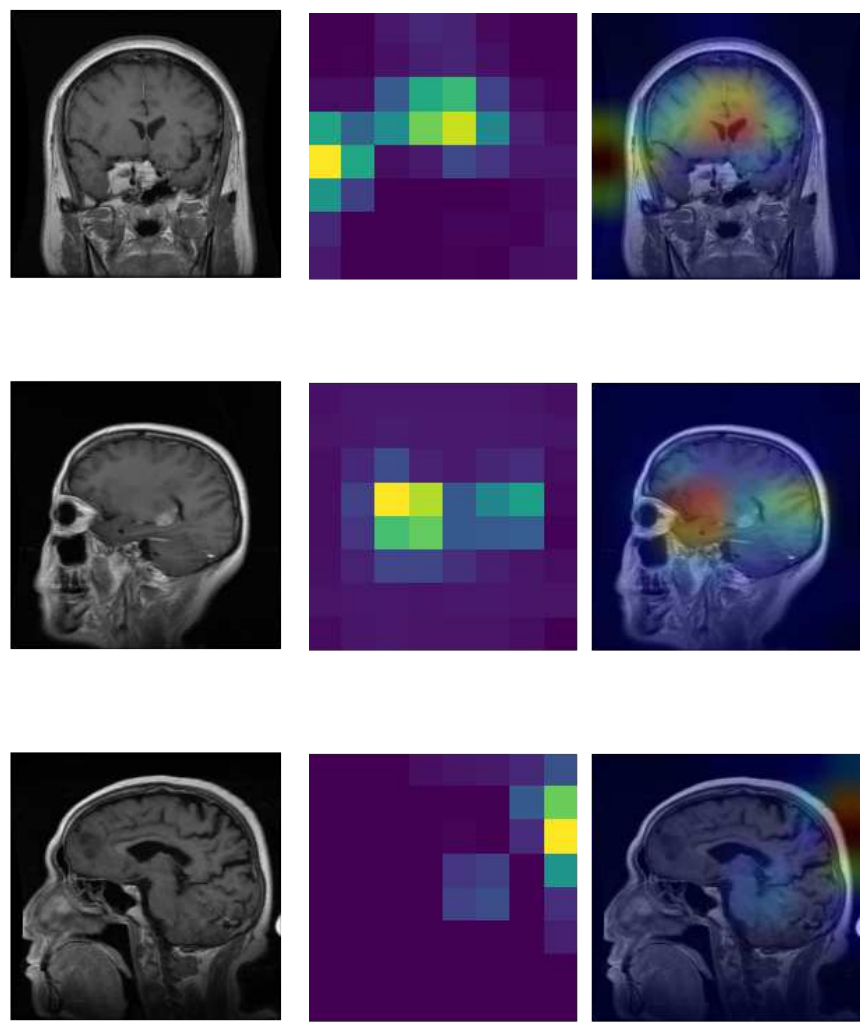

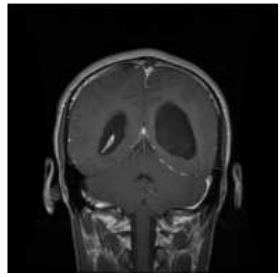

(a) Original

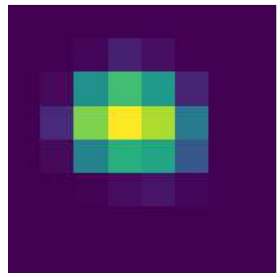

(b) Proposed model
Fig. 7: Class prediction maps on BTDS dataset slices

The results of proposed EEMC model has compared with the popular pre-trained models as shown in Table 5. ResNet50v2 reports the least performance while EfficientNetB4 achieves the best performance in the pretrained models. The proposed EEMC model outperforms existing pre-trained models with $97.65 \%$ accuracy. Moreover, our model exhibits an improved performance of $1 \%$ than EfficientNet models and $2 \%$ than other models. 
Table 5: Comparision with pre-trained models

\begin{tabular}{|l|cccc|}
\hline Model & Accuracy & Precision & Recall & Jaccard \\
\hline GoogleNet & 95.14 & 94.54 & 95.09 & 90.06 \\
EfficientNet2 & 96.34 & 95.58 & 96.48 & 92.33 \\
EfficientNet4 & 96.54 & 96.12 & 96.34 & 92.73 \\
ResNet50v2 & 84.59 & 81.95 & 81.63 & 72.65 \\
MobileNet & 95.99 & 95.15 & 96.22 & 91.72 \\
EEMC & 97.65 & 97.33 & 97.34 & 94.81 \\
\hline
\end{tabular}

Table 6: Comparision with state-of-the-art models

\begin{tabular}{|l|c|c|}
\hline Ref. & \# Parameters & Accuracy \\
\hline Anraki et al. $[13]$ & $2.2 \mathrm{M}$ & 94.20 \\
Deepak et al. $[16]$ & $21.8 \mathrm{M}$ & 97.10 \\
Venkateswarlu et al. $[22]$ & $5.5 \mathrm{M}$ & 97.52 \\
EEMC & $11.5 \mathrm{M}$ & 97.65 \\
\hline
\end{tabular}

We have considered accuracy to compare the proposed model with state-of-the-art models. Table 6 lists out the accuracy of state-of-the-art models along with the proposed model. Our model exhibits $0.5 \%$ improvement than Deepak et al. and similar performance like Venkateswarlu et al. model. This table also lists out the number of trainable parameters and our model uses 11.5 M parameters.

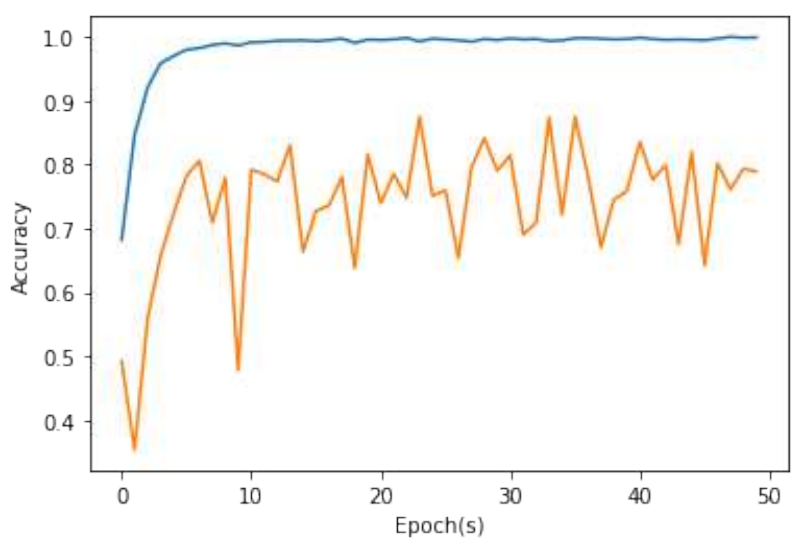

Fig. 8: Training vs validation accuracy

\subsection{Evaluation of proposed model on CPM dataset}

We have included the performance evaluation of the proposed EEMC model on the CPM dataset in this section. The training and validation accuracy of the proposed EEMC model can be visualized from Fig. 8. Table 7 list out stratified five-fold cross-validation results of the proposed EEMC model. Our model has achieved the best performance in the second fold with $96.85 \%$ accuracy. It is observed that our model has experienced the least performance in the third fold. Further, our
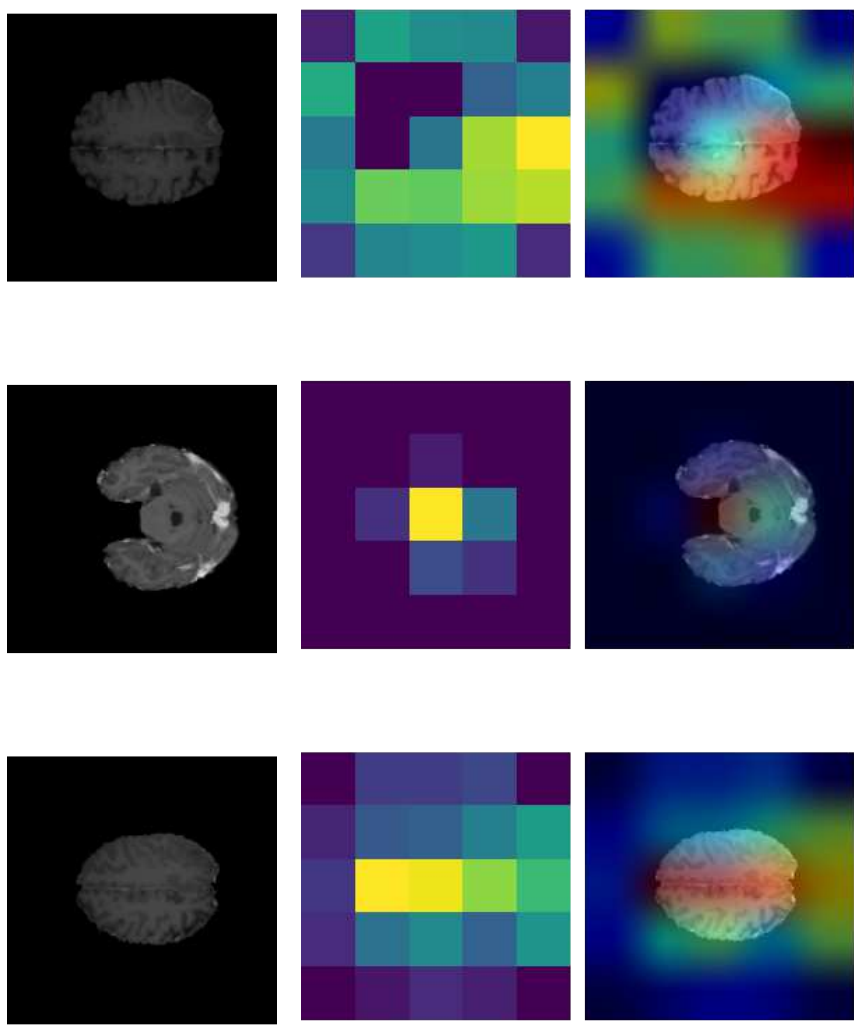

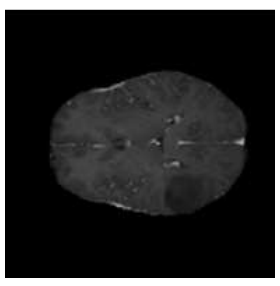

(a) Original

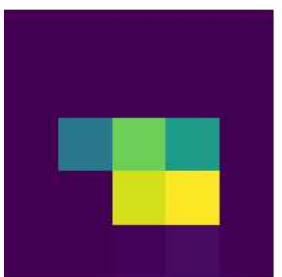

(b) Proposed model

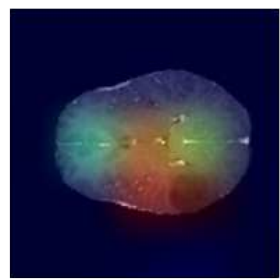

Fig. 9: Class prediction maps on CPM dataset slices

model attains a mean accuracy of $95.84 \%$ with a 0.99 deviation.

Table 7: Stratified five-fold cross-validation

\begin{tabular}{|c|cccc|}
\hline Fold & Accuracy & Precision & Recall & Jaccard \\
\hline 1 & 96.62 & 94.44 & 97.4 & 91.98 \\
2 & 96.85 & 95.54 & 96.86 & 92.64 \\
3 & 94.54 & 94.27 & 92.18 & 86.74 \\
4 & 95.1 & 93.37 & 96.65 & 90.24 \\
5 & 96.11 & 94.68 & 96.09 & 91.14 \\
\hline Mean & 95.84 & 94.46 & 95.84 & 90.55 \\
Stddev & 0.99 & 0.78 & 2.1 & 2.31 \\
\hline
\end{tabular}

Our model detects the exact location of the tumor for the skull-stripped brian tumor images as shown in Fig. 9. However, the proposed model results in the wrong prediction when there is a less significant tumor 
as shown in the first three rows in Fig. 9. Table 8 compares the performance of the proposed EEMC model with the popular pre-trained models. In this case, EfficientNet2 shows the best performance in the existing pre-trained model. The proposed EEMC model exists a similar performance as EfficientNet2 with $95.84 \%$ accuracy. Similarly, Table 6 lists out the accuracy of stateof-the-art models along with the proposed model. Our model exhibits $1 \%$ improvement than Venkateswarlu et al. and similar performance as Deepak et al. model. This table also lists out the number of trainable parameters and our model uses $11.5 \mathrm{M}$ parameters.

Table 8: Comparision with pre-trained models

\begin{tabular}{|l|cccc|}
\hline Model & Accuracy & Precision & Recall & Jaccard \\
\hline GoogleNet & 90.29 & 88.45 & 90.33 & 81.09 \\
ResNet50v2 & 87.97 & 85.63 & 87.85 & 76.53 \\
EfficientNet2 & 95.23 & 93.8 & 95.27 & 89.52 \\
EfficientNet4 & 94.52 & 92.51 & 95.87 & 88.73 \\
MobileNet & 89.92 & 89.92 & 89.92 & 81.86 \\
EEMC & 95.84 & 94.46 & 95.84 & 90.55 \\
\hline
\end{tabular}

Table 9: Comparision with state-of-the-art models

\begin{tabular}{|l|c|c|}
\hline Ref. & \# Parameters & Accuracy \\
\hline Anraki et al. $[13]$ & $2.2 \mathrm{M}$ & 91.68 \\
Deepak et al. $[16]$ & $21.8 \mathrm{M}$ & 95.19 \\
Venkateswarlu et al. $[22]$ & $5.5 \mathrm{M}$ & 94.85 \\
EEMC & $11.5 \mathrm{M}$ & 95.84 \\
\hline
\end{tabular}

\subsection{Discussion}

In this work, we have proposed an ensemble EfficientNet and mixed convolution for three-class brain tumor classification. The implementation of a generalized model is a critical task for deep learning due to the lack of huge datasets. From the results, the following things can be observed.

1. EfficientNetB4 performs best on BTDS dataset and EfficientNetB2 exhibits best result on CPM dataset.

2. The proposed EEMC model outperforms the existing model on the BTDS dataset and exhibits similar performance than existing models.

3. Our model learns semantic features of the brain and detects accurate tumor location while predicting tumor class.

\section{Conclusion}

The three-class classification becomes a critical research task in computer-aided brain tumor diagnosis. The implementation of a generalized model is a critical task for deep learning due to the lack of huge datasets. In this paper, we have proposed an ensemble EfficientNet and mixed convolution model for three-class brain MR image classification. The proposed ensemble model has utilized EfficientNetB2 for the feature extraction. Then, we have proposed a mixed convolution block to capture semantic features of the brain. The mixed convolution block consists of two sub-blocks including separable convolution and residual convolution. The proposed model has devised global average pooling and gaussian dropout layer to avoid model overfitting. In our experiments, two publicly available datasets are considered for the evaluation of the proposed EEMC model. Our ensemble model outperforms the existing model on the BTDS dataset and exhibits similar performance to existing models. We have achieved an accuracy of $97.65 \%$ and $95.84 \%$ on BTDS and CPM datasets, respectively. Further, our model learns semantic features of the brain and detects accurate tumor location while predicting tumor class.

\section{Compliance with ethical standards}

Conflict of interest The authors declare that there is no conflict of interest regarding the publication of this paper.

Ethical approval This article does not contain any studies with human participants or animals performed by any of the authors.

Informed consent Informed consent was obtained from all individual participants included in the study.

\section{References}

1. A. El-Sayed, El-Dahshan, H.M. Mohsen, K. Revett, A.B.M. Salem, Expert Systems with Applications 41(11), $5526(2014)$

2. Y. Zhang, X. Chen, T. Zhan, Z. Jiao, Y. Sun, Z. Chen, Y. Yao, L. Fang, Y. Lv, S. Wang, IEEE Access 4, 5937 (2016)

3. D.R. Nayak, R. Dash, B. Majhi, S. Wang, Computers and Electrical Engineering 68, 366 (2018)

4. T. Kaur, B.S. Saini, S. Gupta, Biocybernetics and Biomedical Engineering 38(2), 409 (2018)

5. S. K, M. Elhoseny, L. S K, I. M, V. RM, M. A. Elsoud, M. Alkhambashi, Concurrency and Computation: Practice and Experience 32(1), e4887 (2020)

6. H.M. School. Whole brain atlas (2019). URL http://www.med.harvard.edu/AANLIB/. Accessed June 2019

7. S. Lu, Z. Lu, Y.D. Zhang, Journal of Computational Science 30, 41 (2019)

8. M. Havaei, A. Davy, D. Warde-Farley, A. Biard, A. Courville, Y. Bengio, C. Pal, P.M. Jodoin, H. Larochelle, Medical Image Analysis 35, 18 (2017)

9. R.L. Kumar, J. Kakarla, B.V. Isunuri, M. Singh, Multimedia Tools and Applications 80(9), 13429 (2021) 
10. J. Kakarla, B.V. Isunuri, K.S. Doppalapudi, K.S.R. Bylapudi, International Journal of Imaging Systems and Technology (2021)

11. J. Cheng, W. Yang, M. Huang, W. Huang, J. Jiang, Y. Zhou, PLoS ONE 11(6) (2016)

12. A. Gumaei, M.M. Hassan, M.R. Hassan, A. Alelaiwi, G. Fortino, IEEE Access 7, 36266 (2019)

13. A.K. Anaraki, M. Ayati, F. Kazemi, Biocybernetics and Biomedical Engineering 39(1), 63 (2019)

14. Z.N.K. Swati, Q. Zhao, M. Kabir, F. Ali, Z. Ali, S. Ahmed, J. Lu, Computerized Medical Imaging and Graphics 75, 34 (2019)

15. N. Ghassemi, A. Shoeibi, M. Rouhani, Biomedical Signal Processing and Control 57, 101678 (2020)

16. S. Deepak, P. Ameer, Computers in Biology and Medicine 111, 103345 (2019)

17. J. Cheng, BTDS (accessed June 2019). URL https://figshare.com/articles/brain_tumor_ dataset $/ 1512427$

18. S. Bakas, CPM-RadPath (accessed June 2020). URL http://miccai2020data.eastus.cloudapp.azure.com/CPMRadPath_2020_Training_Data/

19. M. Tan, Q. Le, in International Conference on Machine Learning (PMLR, 2019), pp. 6105-6114

20. K. He, X. Zhang, S. Ren, J. Sun, in Proceedings of the IEEE conference on computer vision and pattern recognition (2016), pp. 770-778

21. A.G. Howard, M. Zhu, B. Chen, D. Kalenichenko, W. Wang, T. Weyand, M. Andreetto, H. Adam, arXiv preprint arXiv:1704.04861 (2017)

22. B.V. Isunuri, J. Kakarla, Concurrency and Computation: Practice and Experience p. e6541 (2021) 